\title{
Orientações de enfermagem no cuidado ao lactente com choro excessivo: revisão integrativa
}

\author{
Nursing guidelines in the care of infants with excessive crying: integrative review
}

Pautas de enfermería para el cuidado de bebés con grito excesivo: revisión integrativa

Adriana Paiva Camargo Saraiva ${ }^{1 *}$, Linda Inêz Alves da Silva ${ }^{1}$, Sandiely Lorrainy de Carvalho Souza1.

\begin{abstract}
RESUMO
Objetivo: Investigar métodos terapêuticos que a equipe de enfermagem possa recomendar para pais e familiares a fim de minimizar o choro excessivo em lactentes. Métodos: Revisão integrativa, realizada em março de 2020, produzida a partir da estratégia PICOS, nas plataformas Biblioteca Virtual em Saúde (BVS), Literatura Internacional em Ciências da Saúde (PUBMED) e Cochrane, através da inclusão de artigos disponíveis online e gratuitos, que retratam a temática vigente, em inglês e português, publicados entre 2016 e 2020. Resultados: Foram encontrados 180 artigos nas bases de dados, excluídos 120 após a aplicação dos critérios de inclusão/exclusão, selecionados 60 para serem lidos na íntegra e, ao final, 11 artigos foram incluídos nesta revisão. Considerações finais: Acupuntura, quiropraxia e massagem terapêutica sobressaíram nas pesquisas, embora nem sempre os estudos analisados tenham chegado a resultados similares. A massagem foi recomendada, embora o aconselhamento parenteral seja a forma mais viável de consolar o choro e aliviar a cólica no lactente.
\end{abstract}

Palavras-chave: Choro excessivo, Cólica Infantil, Terapias.

\begin{abstract}
Objective: Investigate therapeutic methods that the nursing staff may recommend for parents and families in order to minimize excessive crying in infants. Methods: Integrative review, carried out in March 2020, produced from the PICOS strategy, on the Virtual Health Library (VHL), International Literature on Health Sciences (PUBMED) and Cochrane platforms, through the inclusion of articles available online and free, which portray the current theme, in English and Portuguese, published between 2016 and 2020. Results: 180 articles were found in the databases, 120 were excluded after applying the inclusion/exclusion criteria, 60 were selected to be read in full and, in the end, 11 articles were included in this review. Final considerations: Acupuncture, chiropractic care and therapeutic massage stood out in the surveys, although the analyzed studies did not always reach similar results. Massage was recommended, although parenteral counseling is the most viable way to console crying and alleviate colic in the infant.
\end{abstract}

Key words: Excessive crying, Infantile colic, Therapies.

\section{RESUMEN}

Objetivo: Investigar métodos terapéuticos que el personal de enfermería pueda recomendar a los padres y las familias con el fin de minimizar el llanto excesivo en los bebés. Métodos: Revisión integradora, realizada en marzo de 2020, producida a partir de la estrategia PICOS, sobre la Biblioteca Virtual en Salud (BVS), Literatura Internacional en Ciencias de la Salud (PUBMED) y plataformas Cochrane, mediante la inclusión de artículos disponibles en línea y gratuitos, que retratan la actualidad. tema, en inglés y portugués, publicado

1 Universidade do Estado do Pará (UEPA), Conceição do Araguaia - PA. *E-mail: adriana.saraiva@uepa.br SUBMETIDO EM: 9/2021 
entre 2016 y 2020. Resultados: Se encontraron 180 artículos en las bases de datos, 120 fueron excluidos luego de aplicar los criterios de inclusión / exclusión, 60 fueron seleccionados para ser leídos íntegramente y, finalmente, se incluyeron 11 artículos en esta revisión. Consideraciones finales: En las encuestas destacaron la acupuntura, la atención quiropráctica y el masaje terapéutico, aunque los estudios analizados no siempre alcanzaron resultados similares. Se recomendó el masaje, aunque el asesoramiento parenteral es la forma más viable de consolar el llanto y aliviar los cólicos en el bebé.

Palabras clave: Llanto excesivo, Cólico infantil, Terapias.

\section{INTRODUÇÃO}

A Cólica do Lactente (CL) pode ser definida mediante os critérios de Wessel, por episódios de choro incontrolável constantes durante a noite ou no mesmo horário em pelo menos três horas por dia, durante pelo menos três dias por semana, em pelo menos três semanas. Acomete cerca de uma em cada cinco crianças com menos de três meses e é caracterizada por episódios de paroxismo, irritabilidade, agitação, ausência de ganho de peso, distensão abdominal, contração das pernas, chutes, punhos apertados dentre outras manifestações de dor (HOLM LV, et al., 2018).

Ainda não há uma causa específica para a cólica do lactente, contudo, originalmente a palavra "cólica" significa "intestino grosso", que está relacionada com as possíveis disfunções no sistema digestivo, tais como a superprodução de gás intestinal, contração intestinal intensa, falta de comunicação entre cérebro e intestino, hipersensibilidade à proteína do leite de vaca, deficiência transitória de lactase e ligação materno-infantil negativa ou inadequada (HOLM LV, et al., 2018).

Para Gordon M, et al. (2018), a má absorção de carboidratos leva à fermentação colônica e a um aumento nos níveis de gás hidrogênio que distende o cólon causando dor, enquanto as pressões osmóticas geradas pela lactose e ácido lático no cólon causam um influxo de água, levando a uma maior distensão do intestino. Darque AA, et al. (2020), demonstraram que logo após o nascimento, os bebês que apresentam maior reatividade aos estímulos sensoriais, especialmente olfatórios, possuem maior tendência para cólica. Essa sensibilidade precoce pode explicar o comportamento de choro desconsolado nas seis primeiras semanas de vida.

A colonização do trato gastrintestinal (TGI) do RN e lactente dependem principalmente do tipo de parto e alimentação. Estudos afirmam que a CL pode estar relacionada à grande incidência de cesariana, pois, 0 contato com a microbiota vaginal da mãe no parto normal auxilia no fortalecimento da imunidade e no processo de formação da flora bacteriana do RN, o que não ocorre no parto cesáreo (MORAIS MB, 2016).

Além disso, devido à imaturidade do TGI do lactente, especialmente do reflexo da deglutição, da menor atividade de lactase e o padrão imaturo de motilidade do TGl, a alimentação do lactente torna-se um desafio, porque fica susceptível a refluxo gastroesofágico, constipação intestinal e intolerância alimentar (MORAIS $M B, 2016$ ). A hipersensibilidade associada à alergia alimentar ou mesmo com o tabagismo materno, devido ao aumento dos níveis plasmáticos e intestinais de motilina também são causadores da CL (MOREIRA AC, et al., 2019).

Outra possibilidade é a de que o sistema circadiano que é maturado durante os três primeiros meses de vida e cumpre importante função no sistema nervoso central, que é regulado pelo hipotálamo, afeta as atividades fisiológicas como horário do sono, temperatura corporal, alimentação e produção de hormônios, como a melatonina, e esteja associado às cólicas e o choro excessivo infantil (HALPERN R e COELHO R., 2016).

Causas psicossociais, como tensão familiar, ansiedade parental ou interação inadequada também são veementemente defendidas (MOREIRA AC, et al., 2019). Atrelado ao fato dos cuidadores muitas vezes não saberem lidar com a situação, têm-se os riscos para a depressão materna, estresse parental e prejuízos ao neonato a curto, médio e a longo prazo, como distúrbios do sono, problemas no relacionamento familiar, e 
até mesmo distúrbios psicológicos em vidas futuras de bebês com cólica como perturbações do sono, as capacidades de processamento sensorial, falta de concentração, hiperatividade e birras (HOLM LV, et al., 2018).

Dessa forma, Darque AA, et al. (2020), afirmam que o choro prolongado e inexplicável do bebê é um dos primeiros desafios mais comuns para os pais e profissionais que Ihes prestam suporte. Com o uso de práticas integrativas e complementares é possível evitar tratamentos invasivos e desnecessários e assistir ao paciente de forma holística, consistente e humanizada. Assim, entende-se que os(as) enfermeiros(as) possuem condições de explorar diversas modalidades terapêuticas no exercício de suas atribuições, podendo implementar e recomendar o uso de terapias que venha alentar mecanismos naturais para prevenção e recuperação da saúde de forma resoluta e humanizada.

No contexto de queixa de CL é imprescindível que orientem adequadamente os cuidadores para minimizar o sofrimento do paciente e de seus familiares, e acredita-se que o uso de terapias alternativas e complementares para o alívio da CL, auxiliem nesse propósito e no crescimento e desenvolvimento saudável do infante (BARR RG, 2017). Portanto, o objetivo dessa pesquisa foi buscar na literatura científica, reunir e analisar estudos sobre os métodos terapêuticos que a equipe de enfermagem possa recomendar aos cuidadores para a redução da cólica e choro do lactente.

\section{MÉTODOS}

Trata-se de uma revisão integrativa, na qual utilizou-se a estratégia PICOS que representa um acrônimo para Paciente, Intervenção, Comparação, "Outcomes" (desfecho) e Tipo de estudo, para a construção da pergunta de pesquisa e busca de evidências (GALVÃO TF e PEREIRA MG, 2014). O trabalho teve a seguinte subdivisão: identificação do problema (pergunta norteadora); definição dos critérios de inclusão e exclusão (seleção da amostra); elucidação das informações a serem extraídas dos trabalhos revisados; análise das informações; discussão (interpretação dos resultados) e apresentação da revisão (conclusão).

Como questão norteadora para esta pesquisa: Há evidências científicas disponíveis sobre terapia para choro excessivo ou cólica do lactente que podem fomentar as orientações de enfermagem?

Os critérios de inclusão foram: artigos disponíveis online e gratuitos, que retratam a temática vigente, publicados em inglês e português entre os anos de 2016 a 2020. Os critérios de exclusão foram: artigos que não estavam disponíveis na íntegra, bem como aqueles em que os autores realizaram suas pesquisas utilizando intervenção farmacológica.

A pesquisa eletrônica ocorreu em Março de 2021, nas seguintes plataformas de pesquisa: Biblioteca Nacional de Medicina dos Estados Unidos (PubMed), Cochrane Library (Abrange uma coleção de bancos de dados em medicina e outras especialidades da área de saúde) e na Biblioteca Virtual em Saúde (BVS), a qual contempla várias bases de dados, dentre elas a Medical Literature and Retrieval System online (Medline), Literatura Latino Americana em Ciências da Saúde (Lilacs), e Base de Dados de Enfermagem (BDENF). As palavras-chave/ descritores utilizados na busca combinada foram: infant colic (CL), excessive crying (choro excessivo) e therapy (terapia), associados aos termos booleanos AND e OR, formando a seguinte combinação: infant colic OR excessive crying AND therapy. As informações obtidas dos estudos selecionados foram: autor/ano, base de dados, objetivo, tipo de estudo, amostra, intervenção e conclusão.

\section{RESULTADOS}

A pesquisa na plataforma PubMed, com a associação dos descritores: excessive crying OR infant colic AND therapy, resultou em 66 artigos; no portal regional da BVS, a busca resultou em 109 artigos com a combinação excessive crying AND therapy; na base de dados Cochrane, utilizando a combinação excessive crying AND therapy, resultou em 5 artigos. Após aplicação dos critérios de inclusão e exclusão, 11 artigos foram incluídos (Figura 1). 
Figura 1 - Fluxograma da seleção dos estudos incluídos na revisão integrativa.

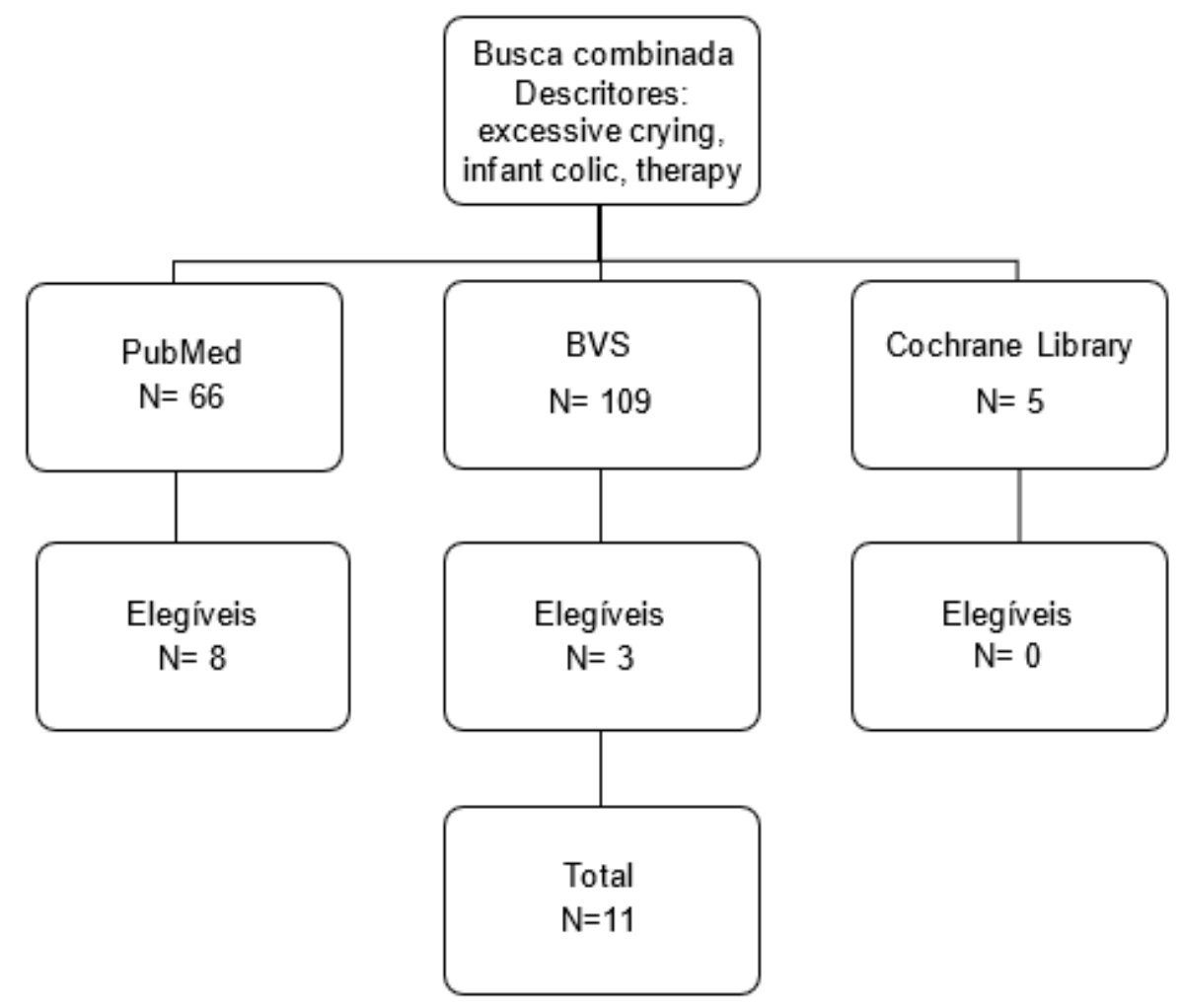

Fonte: Saraiva APC, et al., 2021.

No que se refere ao ano de publicação, o ano com maior número de estudos publicados foi 2018, com 6 publicações. Com relação à metodologia empregada, 3 artigos são de Ensaio Clínico Randomizado (ECR) ou protocolo de ECR, 7 são revisões sistemáticas e uma revisão integrativa. O Quadro 1, mostra os aspectos dos ensaios clínicos randomizados selecionados para esse estudo. E o Quadro 2, mostra os aspectos dos estudos de revisões integrativas e sistemáticas.

Dos estudos encontrados sobre as medidas de redução do choro excessivo e da CL em lactentes, quatro artigos analisaram a acupuntura, dois sobre massagem e os demais sobre variados meios de tratamento da CL. 
Quadro 1 - Quadro sinóptico com os principais aspectos de cada ensaio ou protocolo de ensaio clínico controlado randomizado, 2020.

\begin{tabular}{|c|c|c|c|c|c|c|}
\hline Autor/ano & $\begin{array}{l}\text { Base de } \\
\text { dados }\end{array}$ & Objetivo & $\begin{array}{l}\text { Tipo de } \\
\text { estudo }\end{array}$ & Amostra & Intervenção & Conclusão \\
\hline $\begin{array}{c}\text { LANDGREN K e } \\
\text { HALLSTRÖM I (2017) }\end{array}$ & $\begin{array}{l}\text { PUBMED } \\
\text { (MEDLINE) }\end{array}$ & $\begin{array}{l}\text { Avaliar e comparar o } \\
\text { efeito de dois tipos de } \\
\text { acup. versus sem acup. }\end{array}$ & ECR & $147 \mathrm{RN}$ & $\begin{array}{l}\text { G1: acup. } \\
\text { mínima LI4; G2: } \\
\text { acup. tradicional } \\
\text { chinesa; G3: } \\
\text { sem acup. }\end{array}$ & $\begin{array}{l}\text { A acup. parece reduzir o choro com } \\
\text { segurança. }\end{array}$ \\
\hline $\begin{array}{l}\text { HOLM LV, et al. } \\
\qquad(2018)\end{array}$ & $\begin{array}{l}\text { PUBMED } \\
\text { (MEDLINE) }\end{array}$ & $\begin{array}{l}\text { Descrever protocolo de } \\
\text { ECR para avaliar a } \\
\text { eficácia do tratamento } \\
\text { quiroprático na Cl. }\end{array}$ & $\begin{array}{l}\text { Protocolo de } \\
\text { ECR }\end{array}$ & $\begin{array}{l}200 \text { cri. } 2 \text { a } 14 \\
\text { semanas }\end{array}$ & $\begin{array}{l}\text { G1: quiropraxia } \\
\text { G2: sem } \\
\text { quiropraxia }\end{array}$ & $\begin{array}{l}\text { Dificuldade de aceitação de pais em } \\
\text { participar do G2 e da logística dos } \\
\text { pais irem até a clínica de quiropraxia. }\end{array}$ \\
\hline $\begin{array}{l}\text { MANSOURI S, et al. } \\
\qquad(2018)\end{array}$ & $\begin{array}{l}\text { PUBMED } \\
\text { (MEDLINE) }\end{array}$ & $\begin{array}{c}\text { Avaliar a massagem } \\
\text { terapêutica na redução } \\
\text { dos sintomas de cólica } \\
\text { em crianças. }\end{array}$ & $\begin{array}{l}\text { Protocolo de } \\
\text { ECR }\end{array}$ & $\begin{array}{l}100 \text { cri. com } \\
\text { id. inferior a } \\
12 \text { semanas. }\end{array}$ & $\begin{array}{l}\text { G1: massagem } \\
\text { G2: balanço }\end{array}$ & $\begin{array}{c}\text { Massagem terapêutica é um método } \\
\text { eficaz. }\end{array}$ \\
\hline
\end{tabular}

Legenda: $\mathrm{ECR}$ = Ensaio Clínico Randomizado; $\mathrm{RN}=$ recém-nascido; $\mathrm{G}=$ grupo; cri=criança; Id=idade; $\mathrm{Acup}$ = acupuntura $\mathrm{Cl}=\mathrm{CL}$.

Fonte: Saraiva APC, et al., 2021. 
Quadro 2 - Quadro sinóptico com os principais aspectos de cada estudo de revisão, 2020.

\begin{tabular}{|c|c|c|c|c|c|c|}
\hline Autor/ano & $\begin{array}{l}\text { Base de } \\
\text { dados }\end{array}$ & Objetivo & $\begin{array}{l}\text { Tipo de } \\
\text { estudo }\end{array}$ & Amostra & Intervenção & Conclusão \\
\hline $\begin{array}{l}\text { CARNES D, } \\
\text { et al. (2018) }\end{array}$ & $\begin{array}{c}\text { BVS } \\
\text { (MEDLINE) }\end{array}$ & $\begin{array}{l}\text { Avaliar o efeito das } \\
\text { intervenções manuais de } \\
\text { terapia para bebês } \\
\text { saudáveis. }\end{array}$ & $\begin{array}{l}\text { Revisão } \\
\text { Sistemática } \\
\text { e } \\
\text { metanálise }\end{array}$ & $\begin{array}{c}7 \text { ECR, } 7 \text { séries de } \\
\text { casos, } 3 \text { estudos de } \\
\text { coorte, } 1 \text { estudo de } \\
\text { avaliação de } \\
\text { serviços e } 1 \text { estudo } \\
\text { qualitativo. }\end{array}$ & Não se aplica. & $\begin{array}{c}\text { Alguns pequenos benefícios foram } \\
\text { encontrados para terapia manual, } \\
\text { relativamente segura. }\end{array}$ \\
\hline $\begin{array}{l}\text { RAMIREZ } \\
\text { MC e } \\
\text { DURÁN KV } \\
(2017)\end{array}$ & $\begin{array}{l}\text { PUBMED } \\
\text { (MEDLINE) }\end{array}$ & $\begin{array}{c}\text { Determinar a eficácia da } \\
\text { massagem para reduzir a } \\
\text { Cl. }\end{array}$ & $\begin{array}{c}\text { Revisão } \\
\text { integrativa }\end{array}$ & 2 ECR com 40 cri. & $\begin{array}{l}\text { Massagem abdominal; } \\
\text { balanço. }\end{array}$ & $\begin{array}{l}\text { Nenhum estudo respondeu a } \\
\text { questão, no entanto, vários } \\
\text { estudos confirmam que a } \\
\text { massagem pode reduzir o tempo } \\
\text { de choro. }\end{array}$ \\
\hline $\begin{array}{l}\text { LEE D, et al. } \\
\qquad(2018)\end{array}$ & $\begin{array}{l}\text { PUBMED } \\
\text { (MEDLINE) }\end{array}$ & $\begin{array}{c}\text { Avaliar evidências clínicas } \\
\text { da segurança e eficácia da } \\
\text { acup. para Cl. }\end{array}$ & $\begin{array}{c}\text { Revisão } \\
\text { sistemática }\end{array}$ & $\begin{array}{c}4 \text { ECR com cri. De } 0 \\
\text { a } 25 \text { semanas }\end{array}$ & Acupuntura & Sem evidências conclusivas. \\
\hline $\begin{array}{l}\text { PERRY R, et } \\
\text { al. (2019) }\end{array}$ & $\begin{array}{c}\text { BVS } \\
\text { (MEDLINE) }\end{array}$ & $\begin{array}{l}\text { Atualizar a síntese de } \\
\text { evidências da literatura de } \\
\text { pesquisa de medicina } \\
\text { complementar e alternativa } \\
\text { sobre } \mathrm{Cl} \text {. }\end{array}$ & $\begin{array}{c}\text { Revisão } \\
\text { sistemática }\end{array}$ & $\begin{array}{c}16 \text { revisões } \\
\text { sistemáticas que } \\
\text { incluíam ECR de } \\
\text { bebês com id. até } 1 \\
\text { ano. }\end{array}$ & $\begin{array}{l}\text { Probióticos; extrato de } \\
\text { erva-doce; manipulação } \\
\text { da coluna; acup.; soja. }\end{array}$ & $\begin{array}{l}\text { A manipulação da coluna } \\
\text { apresenta benefícios para redução } \\
\text { do choro. A aplicação do óleo de } \\
\text { erva doce é o remédio mais } \\
\text { promissor. Já a acupuntura não é } \\
\text { recomendada. }\end{array}$ \\
\hline
\end{tabular}




\begin{tabular}{|c|c|c|c|c|c|c|}
\hline Autor/ano & $\begin{array}{c}\text { Base de } \\
\text { dados }\end{array}$ & Objetivo & $\begin{array}{l}\text { Tipo de } \\
\text { estudo }\end{array}$ & Amostra & Intervenção & Conclusão \\
\hline $\begin{array}{l}\text { ELLWOOD } \\
\text { J, et al. } \\
(2020)\end{array}$ & $\begin{array}{l}\text { PUBMED } \\
\text { (MEDLINE) }\end{array}$ & $\begin{array}{l}\text { Avaliar a eficácia de quatro } \\
\text { abordagens de tratamento } \\
\text { no tempo de choro da } \\
\text { criança, angústia sono e } \\
\text { eventos adversos. }\end{array}$ & $\begin{array}{c}\text { Revisão } \\
\text { sistemática }\end{array}$ & $\begin{array}{l}32 \text { estudos com } \\
\text { lactentes } \\
\text { amamentados }\end{array}$ & $\begin{array}{c}\text { Probióticos; terapias } \\
\text { manuais; simeticona; } \\
\text { inibidores da bomba de } \\
\text { prótons; } \\
\text { aconselhamento aos } \\
\text { pais e avaliação clínica } \\
\text { da mãe e do bebê. }\end{array}$ & $\begin{array}{l}\text { O uso de probióticos apresentou a } \\
\text { maior evidência, seguido por } \\
\text { terapia manual e aconselhamento } \\
\text { parental indicado pelo tempo de } \\
\text { choro. }\end{array}$ \\
\hline $\begin{array}{l}\text { PREVOST } \\
\text { CP, et al. } \\
(2019)\end{array}$ & $\begin{array}{l}\text { PUBMED } \\
\text { (MEDLINE) }\end{array}$ & $\begin{array}{l}\text { Avaliar o uso da terapia } \\
\text { manual para condições } \\
\text { clínicas na população } \\
\text { pediátrica. }\end{array}$ & $\begin{array}{l}\text { Revisão } \\
\text { sistemática }\end{array}$ & 18 estudos & Terapia manual & $\begin{array}{c}\text { Demonstrou pouca evidência } \\
\text { científica para a terapia } \\
\text { manipulativa. }\end{array}$ \\
\hline $\begin{array}{l}\text { MOREIRA } \\
\text { AC, et al. } \\
(2019)\end{array}$ & BVS & $\begin{array}{c}\text { Revisar a eficácia das } \\
\text { intervenções terapêuticas } \\
\text { na Cl. }\end{array}$ & $\begin{array}{l}\text { Revisão } \\
\text { sistemática }\end{array}$ & 17 estudos & $\begin{array}{l}\text { Terapêuticas não } \\
\text { farmacológicas; } \\
\text { terapêuticas } \\
\text { farmacológicas e } \\
\text { terapêuticas } \\
\text { complementares. }\end{array}$ & $\begin{array}{l}\text { O uso de probióticos (Lreuteri DSM } \\
\text { 17938), mostrou } \\
\text { redução do tempo médio de choro } \\
\text { diário. Existe alguma evidência a } \\
\text { favor da fitoterapia à base de } \\
\text { funcho. }\end{array}$ \\
\hline $\begin{array}{l}\text { HJERN A, et } \\
\text { al. (2020) }\end{array}$ & $\begin{array}{l}\text { PUBMED } \\
\text { (MEDLINE) }\end{array}$ & $\begin{array}{l}\text { Avaliar as evidências para } \\
\text { intervenções para CL. }\end{array}$ & $\begin{array}{l}\text { Revisão } \\
\text { sistemática }\end{array}$ & $21 \mathrm{cri}$. & $\begin{array}{l}\text { Acupuntura e } \\
\text { probióticos }\end{array}$ & $\begin{array}{l}\text { Lactobacillus reuteri DSM } 17938 \text { é } \\
\text { um tratamento promissor, mas são } \\
\text { necessários estudos em mais } \\
\text { populações. }\end{array}$ \\
\hline
\end{tabular}

Legenda: $\mathrm{RN}=$ recém-nascido; cri=criança; Acup.= acupuntura; Cl= CL; ECR = Ensaio Clínico Randomizado; Id=idade; Suplem = suplemento/ suplementação. Fonte: Saraiva APC, et al., 2021. 


\section{DISCUSSÃO}

Muitos pais, perante o choro excessivo do lactente, recorrem às Unidades de Saúde, procurando por ajuda profissional. $O$ enfermeiro, que está mais acessível a esse público, deve estar preparado para seguir a conduta adequada e fornecer as orientações necessárias. As evidências apontam para aconselhamento parental, com segurança, massagem, relativamente segura; uso de probióticos e quiropraxia ainda em discussão; e acupuntura com agulha, com mais riscos em relação aos benefícios (MOREIRA AC, et al., 2019; PERRY R, et al., 2019; MANSOURI S, et al., 2018; CARNES D, et al., 2018).

Entre os ECRs dispostos no Quadro 1, o estudo de Landgren K e Hallström I (2017), percebe-se que mesmo abordando a acupuntura como intervenção, aponta para o tratamento padrão como um pilar no atendimento aos bebês com cólica. Assim, se obteve como resultados a redução do choro que segundo os autores, se deve, em parte à origem benigna da cólica que é de evoluir para a cura com o passar dos dias, entretanto, notaram que nos grupos que receberam a acupuntura houve uma redução do choro maior, de modo a satisfazer os pais. Um aspecto importante apontado é que no período em que os lactentes deixaram de ingerir leite de vaca, os pais receberam conselhos por telefone, muitos deixaram de chorar excessivamente e isso levou os autores a concluir que a mudança dietética e o aconselhamento parenteral são fundamentais.

Para Holm LV, et al. (2018), mesmo com a difícil adesão ao tratamento quiroprático, é possível prevenir distúrbios e beneficiar consideravelmente, lactentes e cuidadores. Mansouri S, et al. (2018) concluíram que a massagem reduziu substancialmente os sintomas da cólica e aumentou a duração do sono nos bebês em comparação com o grupo de balanço. Ou seja, a massagem terapêutica é um método eficaz.

O Quadro 2 traz uma variedade de evidências a respeito dos tratamentos para CL e redução do choro. Sabe-se que os pais costumam sacudir o bebê quando este não para de chorar, sendo um fator contributivo para a síndrome do bebê sacudido (MANSOURI S, et al., 2018). Recentemente, com o intuito de reduzir a incidência dessa síndrome, Barr RG (2017), desenvolveu o The Period of PURPLE Crying (o período de chorar até ficar roxo). A palavra PURPLE é um acrônimo das características comuns do choro do lactente nos primeiros meses de vida: pico do choro; imprevisibilidade temporal de crise de choro prolongados; resistência a ser tranquilizado; expressão de dor na ausência de dor; longas crises de choro, e concentração de choro do fim da tarde/começo da noite (BARR RG, 2017).

O aconselhamento é a primeira linha de terapia que pode ser utilizada enquanto se aguarda a CL executar a sua história natural, que consiste em explicar o padrão de choro normal das crianças, incentivando-os a construir a confiança como pais através da amamentação contínua, de terapias e técnicas infantis para acalmar como o casulo, apertar o bebê, posição de lado, fazer shhhh - shhhh, balanço, sucção, ruído branco, manipulação mínima e simular passeio de carro (SARASU IM, et al., 2018).

Com esse conhecimento, os cuidadores são instruídos a seguir os seguintes procedimentos a fim de não sacudir seus bebês: aumentar o contato, caminhar para reduzir o choro; se afastar se o choro tornar-se muito frustrante, deixar o bebê no berço, e acalmar-se; não sacudir ou agredir o bebê (BARR RG, 2017). JamesRoberts I, et al. (2019), desenvolveu um material de aconselhamento para pais e bebês que choram excessivamente, logo, ao ser ofertado para 52 pais, foi associado à redução da frustração dos pais, ansiedade, depressão, relato de choro infantil e contatos com profissionais de saúde e aumento do conhecimento sobre o choro.

Realizar massagem no abdômen com movimentos circulares e no sentido horário, acalmar o bebê no colo, ou no braço em decúbito ventral no intervalo das mamadas, com compressa mornas ou com uso de bolsas térmicas mornas em contato com o abdômen da criança em no máximo 3 a 5 minutos, são medidas que possuem alguma evidência. Como facilita o peristaltismo do intestino e produz um efeito relaxante e um alto grau de conforto do bebê, acredita-se que os efeitos da massagem podem estar relacionados com o acolhimento, aumento da interação mãe-bebê e da redução nos níveis hormonais para o estresse (BRASIL, 2016; RAMIREZ MC e DURÁN LV, 2017; HALPERN R e COELHO R, 2016).

Carnes D, et al. (2018), encontraram evidências moderadas e favoráveis para a redução do tempo de choro em bebês que recebem cuidados de terapia manual (cerca de 1 hora por dia). Quando associada à 
aromaterapia se mostrou benéfica, devido ao efeito antiespasmódico, sedativo e de interação com o bebê, mas alerta para a possível toxicidade do óleo via tópica e inalatória (RAMIREZ MC e DURÁN KV, 2017). Gordon M, et al. (2018), em consonância com Landgren K e Hallstrom L (2017), falam que avaliar o comportamento da criança é crucial para identificar se o choro excessivo é devido à cólica ou ingestão de alimentos alérgicos como o leite de vaca, amendoim, ovos, nozes, trigo, soja e peixe, sendo imprescindível a realização de testes de retirada desses alérgenos para evitar tratamentos desnecessários (Quadro 1).

Em relação às terapias manipulativas, Lee $D$, et al. (2018) afirmam que a acupuntura pode ser eficaz para reduzir os sintomas da cólica, incluindo choro, alimentação e pode estar associada a eventos adversos pequenos, no entanto, nenhum dos estudos investigados teve amostra suficiente e houve diferenças clínicas nos desenhos de estudo e instrumentos utilizados, por isso tornam-se inconclusivos os efeitos da acupuntura na CL. No entanto, vale ressaltar que os ECR obtiveram resultados positivos quanto a diminuição do choro e CL. Prevost CP, et al. (2019) encontraram evidências insuficientes da quiropraxia sobre a CL. Mas, para Perry $\mathrm{R}$, et al. (2019) a manipulação foi classificada como de boa qualidade e baixo risco de polarização com efeito positivo no tempo de choro (Quadro 1).

A suplementação de probióticos em bebês é baseada na teoria de que a cólica se deve à alteração da flora intestinal (SARASU IM, et al., 2018). A revisão sistemática de Hjern A, et al. (2020), aponta que o $L$. reuteri assazona a microbiota intestinal do bebê, sendo um tratamento promissor para a CL, pois, o período de 2 semanas a 3 meses, período típico em que o choro excessivo aumenta e diminui, vem a ser o mesmo que o intestino previamente estéril do bebê é colonizado por bactérias, e com isso, esse processo inicial possa causar desconforto.

Moreira AC, et al. (2019) e Perry R, et al. (2019) corroboram esse achado afirmando ser consistente o uso de probióticos (L. reuteri DSM 17938), havendo redução do tempo médio de choro diário, em regime de prevenção ou tratamento, mas há aumento significativo do choro em bebês alimentados com mamadeira que recebem o L. reuteri, por isso não é recomendado para lactentes alimentados com fórmulas. Ellwood J, et al. (2020), elucidam que a simeticona e inibidores da bomba de prótons ainda não são totalmente seguros para o bebê, mas os probióticos, particularmente $L$. reuteri, foram mais eficazes para reduzir o tempo de choro em crianças amamentadas ( $-25 \mathrm{~min}$ a $-65 \mathrm{~min}$ ao longo de 24 horas) e não houve eventos adversos quanto a utilização deste probiótico, representando o baixo risco para a criança.

Já Sarasu IM, et al. (2018), evidenciam que probióticos geram benefícios, mas são necessários ECR, pois ainda há estudos que sugerem que a melhora com o uso de probióticos pode ser realmente uma parte do curso natural da condição do que o efeito real. Além disso, também foi sugerido a suplementação de probióticos mesmo antes do desenvolvimento de cólica com o intuito de prevenção, porém, não houve evidências significativas. Outras evidências mostram que a microbiota de bebês com sintomas de cólica contém maiores níveis de bactérias aeróbias, e bebês sem sintomas possuem tipos mais variados de microbiota, visto isso, propõe-se que os probióticos podem gerar maior equilíbrio e fornecer uma microbiota intestinal normal reduzindo os sintomas associados a cólica (MORAIS MB, 2016).

Em relação a fitoterapia, preparações que contenham hortelã demonstraram nenhuma evidência, já o óleo de erva doce mostrou algum efeito para reduzir os sintomas da cólica. Sobre o probiótico $L$. reuteri, a maioria dos comentários acerca do uso, revelaram ser eficaz na redução dos sintomas de cólica (PERRY R, et al., 2019).

\section{CONSIDERAÇÕES FINAIS}

Mediante a pesquisa, conclui-se que dentre os métodos terapêuticos evidenciados, a massagem apresentou resultados mais significativos entre os ensaios clínicos randomizados. Embora, os demais métodos como a acupuntura, quiropraxia e suplementação de probióticos venham demonstrando efeitos benéficos na redução do choro e cólica do lactente, ainda não há evidências suficientes para a prática clínica das mesmas. Logo, a principal linha de cuidado é o aconselhamento parental, fundamental para melhorar a qualidade de vida tanto do bebê como dos envolvidos em seu cuidado. Recomenda-se a partir dessa pesquisa 
realizar avaliação do comportamento do lactente para melhor traçar sua conduta diante dos métodos evidenciados e consequentemente ofertar as orientações cabíveis aos cuidadores.

\section{REFERÊNCIAS}

1. BARR RG. O choro e sua importância para o desenvolvimento psicossocial da criança. Enciclopédia sobre o desenvolvimento da primeira infância. Enciclopédia sobre o Desenvolvimento na Primeira Infância, 2017; 15(06): 4166.

2. BRASIL. Protocolo de Atenção Primária à Saúde da Criança. Secretaria de Estado da Saúde do Distrito Federal. Cadernos de Saúde da Criança, 2016; 3: 91. Disponível em: https://aps.saude.gov.br/biblioteca/visualizar/MTIxMA==. Acessado em: 23 de ago. de 2020.

3. CARNES D, et al. Manual therapy for unsettled, distressed and excessively crying infants: a systematic review and meta-analyses. Revista Eletrônica BMJ Open, 2018; 8(1): 1-14.

4. DARQUE AA, et al. Shedding light on excessive crying in babies. Revista Eletrônica Pediatria, 2020; 89(5): 12391244.

5. ELLWOOD J, et al. Comparação de intervenções comuns para o tratamento da CL: uma revisão sistemática de revisões e diretrizes. Revista Eletrônica BMJ Open, 2020; 10(035405).

6. GALVÃO TF, PEREIRA MG. Revisões sistemáticas da literatura: passos para sua elaboração. Revista Eletrônica Epidemiol Serv Saúde, 2014; 23(1):183-184.

7. GORDON M, et al. Dietary modifications for infantile colic. Cochrane Database of Systematic Reviews, 2018; 10(CD011029).

8. HALPERN R, COELHO R. Excessive crying in infants. Revista Eletrônica Pediatria, 2016; 92(3): S40-5.

9. HJERN A, et al. A systematic review of prevention and treatment of infantile colic. Revista Eletrônica Acta Paediatrica, 2020; 109(9): 1733-1744.

10. HOLM LV, et al. The effect of chiropractic treatment on infantile colic: study protocol for a single-blind randomized controlled trial. Revista Eletrônica Chiropr Man Therap, 2018; 26:17.

11. JAMES-ROBERTS IST, et al. Um pacote de apoio para pais de bebês com choro excessivo: estudo de desenvolvimento e viabilidade. Revista Eletrônica Health Technol Assess, 2019; 23 (56).

12. LANDGREN K, HALLSTRÖM I. Effect of Minimal Acupuncture for Infantile Colic: A Multicentre, Three-Armed, SingleBlind, Randomised Controlled Trial (ACU-COL). Revista Eletrônica Acupunct Med, 2017; 35(3): 171-179.

13. LEE D, et al. Acupuncture for Infantile Colic: A Systematic Review of Randomised Controlled Trials. Evidence-Based Complementary and Alternative Medicine, 2018; (7526234): 1-11.

14. MANSOURI S, et al. A placebo-controlled clinical trial to evaluate the effectiveness of massaging on infantile colic using a random-effects joint model. Pediatric Health Medicine and Therapeutics, 2018; 9: 157-163.

15. MORAIS MB. Signs and symptoms associated with digestive tract development. Revista Eletrônica Pediatria, 2016; 92(3 Suppl 1): S46-56.

16. MOREIRA AC, et al. Tratamento da CL: uma revisão baseada na evidência. Revista Eletrônica Port Med Geral Fam, 2019; 35(5): 367-80.

17. PERRY R, et al. An overview of systematic reviews of complementary and alternative therapies for infantile colic. Systematic Reviews, 2019; 8:271.

18. PREVOST CP, et al. Manual therapy for the pediatric population: a systematic review. BMC Complementary and Alternative Medicine, 2019; 19(1): 60.

19. RAMIREZ MC, DÚRAN KV. Efectividad del masaje en el área abdominal para la reducción de los cólicos del lactante. Revista Electrónica Enfermería Actual, 2017; 32:1409-4568.

20. SARASU JM, et al. Infantile Colic: An Update. Revista Eletrônica Indian Pediatrics, 2018; 55(11): 979-987. 